03,08

\title{
Первопринципные термодинамические характеристики образования кислородных вакансий и примесных центров бора, углерода и азота в анатазе
}

\author{
(С) В.П. Жуков, И.Р. Шеин
}

Институт химии твердого тела УрО РАН, Екатеринбург, Россия

E-mail: Zhukov@ihim.uran.ru

(Поступила в Редакцию 11 мая 2017 г.

В окончательной редакции 21 июня 2017 г.)

Первопринципным методом присоединенных проекторных волн (PAW) выполнены расчеты электронной зонной структуры диоксида титана со структурой анатаза, допированного бором, азотом, углеродом. Установлены термодинамические характеристики образования примесных центров, такие как энергия предпочтения междоузельной позиции, энергия окисления примеси, энергия образования кислородных вакансий. Показано, что в условиях термодинамического равновесия стабильной является междоузельная позиция атомов бора, тогда как атомы углерода, в зависимости от давления кислорода, могут занимать и междоузельные позиции и позиции замещения атомов кислорода, а атомы азота замещают кислородные атомы. Показано, что присутствие кислородных вакансий способствует термодинамической стабильности атомов углерода и азота. Полученные плотности электронных состояний соответствуют данным ЭПР-спектроскопии, свидетельствующим о нахождении спин-поляризованных электронов в состояниях кислородной вакансии.

Исследование проведено при финансовой поддержке в рамках проекта 15-17-3-44 Президиума УрО РАН. Расчеты выполнялись на кластере URAN Института математики и механики УрО РАН.

DOI: 10.21883/FTT.2018.01.45286.150

\section{1. Введение}

Диоксид титана, полупроводник со структурой анатаза и шириной запрещенной щели $\sim 3.2 \mathrm{eV}$, является одним из важнейших современных материалов; он широко используется благодаря высокой стабильности, низкой стоимости, наличию фотокаталитической активности в ультрафиолетовой части солнечного света, сверхсмачиваемости водой и ряду других полезных свойств. Одним из перспективных направлений применения анатаза является создание на его основе эффективных фотокатализаторов обеззараживания воды от химических загрязнителей и болезнетворных бактерий, а также для получения водорода методом фотолиза воды [1-7]. Эффективность фотокатализатора существенно зависит от тех стадий фотокатализа, результатом которых является поглощение кванта света и появление электрон-дырочных пар. Недостатком анатаза как катализатора является то, что его поглощение приходится в основном на ультрафиолетовую часть спектра, тогда как видимая часть, которая содержит более $90 \%$ энергии солнечного света, остается неиспользованной. Поэтому в последние годы проводятся обширные физико-химические исследования, направленные на создание на основе анатаза таких фотокатализаторов, которые имели бы поглощение в видимой части спектра, но при этом обладали бы высокой подвижностью электронов и дырок, генерируемых в процессе фотокатализа, и малой скоростью их рекомбинации. Одним из направлений разработки эффективных фотокатализаторов является поиск таких методов их допирования, которые за счет появления примесных центров в запрещенной щели или сдвига края фундаментального поглощения приводили бы к улучшению указанных свойств. Было, в частности, показано, что заметного повышения поглощения света удается достичь за счет допирования анатаза углеродом [8-11], азотом [12-15], а в некоторых случаях и бором [16-20], что сопровождается повышением фотокаталитической активности в ряде реакций. Поэтому исследованию структуры и свойств примесных центров бора, углерода или азота в анатазе, их влянию на свойства материала было посвящено большое число как экспериментальных, так и теоретических работ.

Поскольку рентгеновские дифракционные методы исследования кристаллической структуры мало чувствительны к присутствию примесей $2 p$-элементов, основными методами исследования примесных центров были рентгеновская эмиссионная, ЯМР- и ЭПР-спектроскопии. Также был проведен ряд исследований с использованием колебательной и оптической спектроскопии. Интерпретация таких спектров обычно производится путем их сопоставления со спектрами родственных соединений, что позволяет идентифицировать нахождение примесных атомов в структуре, но остаются проблемы, относящиеся к местам локализации примесных атомов, их магнитному состоянию и степени окисления. Для получения такой информации в ряде работ были привлечены первопринципные методы расчета электронной зонной структуры и свойств соединений. Обзоры приложений первопринципных методов были недавно 
сделаны в $[21,22]$, поэтому ниже мы остановимся только на некоторых работах $[17,18,23-26]$, в которых при изучении свойств материалов было учтено искажение кристаллической структуры в присутствии примесей. В данных работах на основе расчетов зонной структуры была дана интерпретация спектров ЭПР-соединений, изучены локальные деформации кристалла вблизи примесных атомов бора, углерода и азота, выявлены наиболее вероятные места локализации примесных атомов и рассчитаны энергии образования примесей и вакансий в кислородной подрешетке.

Полученные в теоретических работах результаты составляют базу для интерпретации экспериментальных данных, но не лишены недостатков. В частности, подвержены критике использованные в таких работах методы оценки химического потенциала атомов, которые нужны для расчета энергии образования дефектов. Основная проблема в оценке химпотенциалов заключается в том, что в реальных процессах синтеза поставщиком примесных атомов обычно являются не какие-то вещества с простой структурой, например $\mathrm{B}_{2} \mathrm{H}_{6}$ [24], а сложные органические соединения - прекурсоры, поэтому химпотенциалы примесных атомов могут заметно отличаться от принятых в расчетах. Примером результата, не имеющего экспериментального подтверждения является, сделанный на основе расчетов энергии образования дефектов, вывод работы [23] о том, что преимущественными местами локализации атомов углерода, введенных в анатаз, являются позиции в подрешетке атомов титана.

Достаточно надежными можно считать лишь методы оценки химпотенциалов двухатомных молекул, например кислорода или азота $[23,26]$, которые сочетают первопринципные расчеты полной энергии молекул и учет зависимости химпотенциала от давления и температуры. Ниже мы покажем, что, опираясь только на такие потенциалы и не используя химпотенциалы атомов допирующего элемента, на базе первопринципных расчетов электронной зонной структуры можно вычислить некоторые термодинамические характеристики примесных центров. Такие расчеты, проведенные нами для примесей бора, углерода и азота, позволили сделать выводы о местах локализации внедряемых атомов в зависимости от условий синтеза, оценить стабильность внедренных атомов в кислородном окружении и зависимость термодинамических характеристик от присутствия вакансий в кислородной подрешетке.

Заметим, что в первопринципных расчетах электронной структуры нами был использован более совершенный, чем в $[23,26]$, подход, что позволяет оценить степень корректности полученных в предыдущих работах термодинамических характеристик. А именно, мы применяли псевдо-потенциальный метод присоединенных проекторных волн (PAW), реализованный в программном комплексе VASP (Vienna ab initio simulation package, версия 5.2) [27]. Сопоставление метода РAW с использованным в работах $[23,25,26]$ псевдо-потенциальным методом с ультрамягкими псевдо-потенциалами (PW) имеется в работе [28]. Показано, в частности, что метод PAW является точным методом расчета электронной структуры, пригодным для химических соединений с произвольной степенью ионности, тогда как метод PW можно рассматривать как линеаризованную версию метода PAW. Метод PW близок к PAW для металлов или соединений с малой степенью ионности, но может приводить к неточностям для соединений с большой степенью ионности [28].

\section{2. Метод расчета электронной структуры анатаза с примесями бора, углерода, азота}

Моделирование примесных центров проводилось с использованием сверхъячейки, полученной путем двукратных трансляций примитивной ячейки анатаза $\mathrm{Ti}_{2} \mathrm{O}_{4}$ вдоль каждого кристаллографического направления, т.е. орторомбической ячейки $\mathrm{Ti}_{16} \mathrm{O}_{32}$. Расчеты выполнялись как для случая замещения одного атома кислорода примесными атомами, так и для внедрения одного атома в междоузлия. Аналогичным образом, путем удаления одного атома кислорода, моделировалось появление в структуре анатаза кислородной вакансии. При выбранной таким образом сверхъячейке это соответствует концентрации примесных элементов или вакансий равной 3.125 at $\%$

Существенными факторами, которые могут влиять на точность расчетов, являются выбор числа волновых векторов в неприводимой части зоны Бриллюэна (НЧЗБ) и максимального значения энергии плоских волн, используемых для разложения псевдо-волновых функций. Мы проведены расчеты для сетки из 36 волновых векторов в НЧЗБ и максимальной энергии плоских волн равной $400 \mathrm{eV}$. Тесты показали, что данные значения достаточны для получения достаточно точных значений термодинамических характеристик (см. разд. 5). Расчеты проводились в спин-поляризованном варианте, однако для некоторых полученных моделей в процессе самосогласования они сошлись к спин-ограниченному решению (см. разд. 6). Достигнутая во всех случаях точность самосогласования полной энергии была не хуже $10^{-6} \mathrm{eV}$.

Во всех рассматриваемых случаях была проведена оптимизация как объема сверхъячейки, так и атомных координат в соответствии с силами, действующими на атомы (см. описание методики оптимизации в [27]). Минимум полной энергии считался достигнутым, если вариации полной энергии не превышали $1 \mathrm{meV}$. Для оптимизированной таким образом геометрии методом интегрирования по тетраэдрам [27] были построены полные плотности состояний.

\section{3. Геометрия примесных центров}

На рис. 1 показана тетрагональная элементарная ячейка анатаза и места возможного расположения примесных атомов, которые принимались за стартовые в процессе оптимизации кристаллической структуры. 


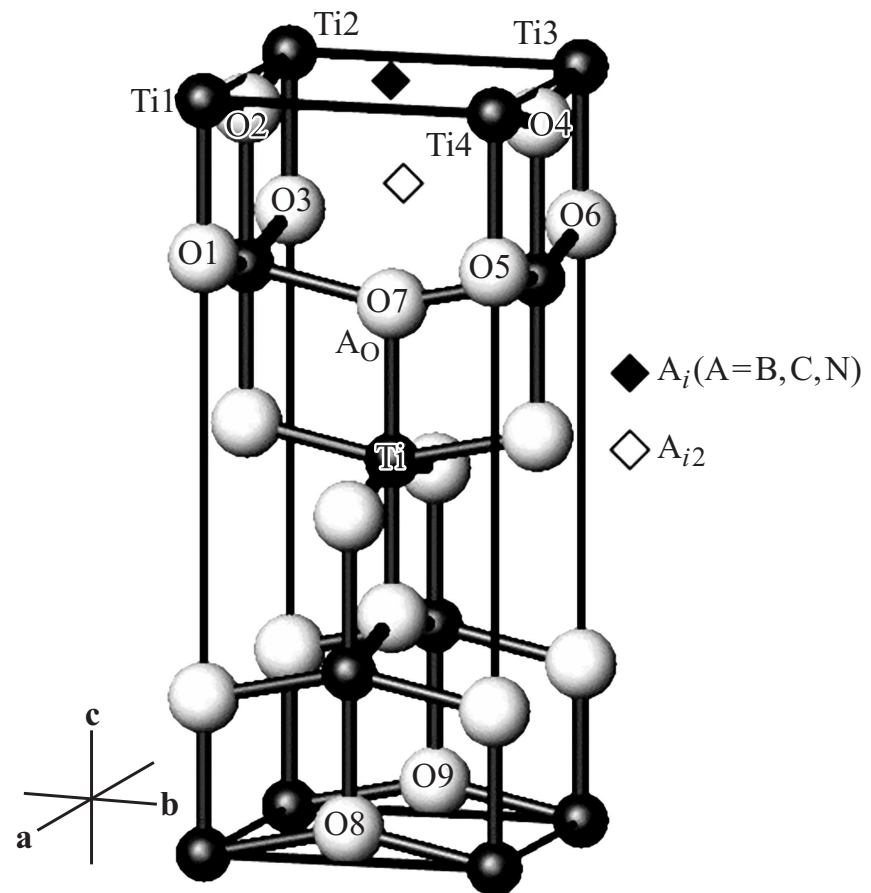

Рис. 1. Тетрагональная элементарная ячейка анатаза. Указаны также позиция замещения атома кислорода или кислородной вакансии, $A_{\mathrm{O}}$, и стартовые местоположения примесных атомов $A_{i}$ и $A_{i 2}, A=\mathrm{B}, \mathrm{C}, \mathrm{N}$.
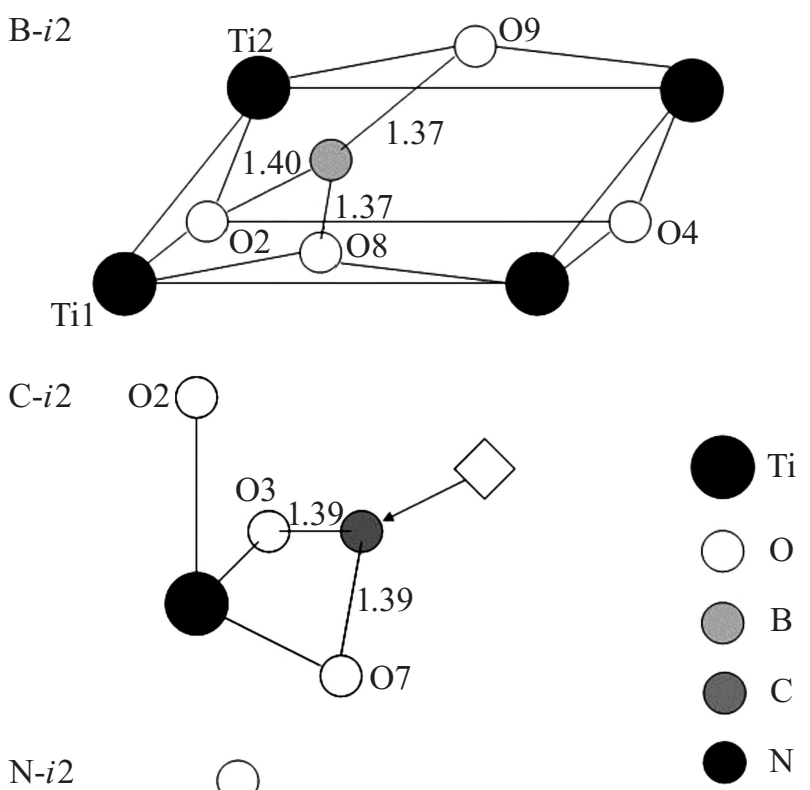

$\mathrm{N}-i 2$

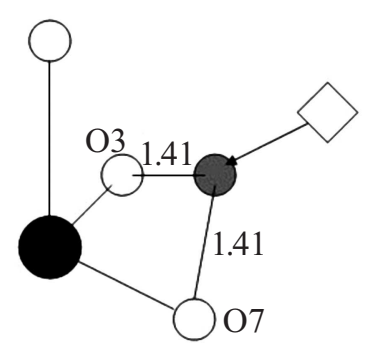

Рис. 2. Схема расположения атомов ближайшего окружения вокруг примесных атомов в позиции $i 2$. Показаны длины связей между соседними атомами, в ед. А̊, и направления сдвига из стартовой позиции.
На рисунке позиция $A_{\mathrm{O}}, A=\mathrm{B}, \mathrm{C}, \mathrm{N}$ внутри треугольника из атомов титана, соответствует примеси замещения или кислородной вакансии. При выборе стартовых междоузельных позиций мы руководствовались соображениями, приведенными в работе [29]. А именно, полагали, что одним из вероятных местоположений внедренного атома может быть наиболее удаленное от атомов исходного кристалла, т.е. позиция $A_{i 2}$ в центре призмы из атомов О1-O2-O3-O4-O5-O6. Но если примесный атом образует химические связи с соседними атомами, то его вероятной позицией может быть и позиция $A_{i}$ в центре квадрата из атомов Ti1-Ti2-Ti3-Ti4, который является также центром сжатого в направлении $c$ кислородного тетраэдра O2-O4-O8-O9. Процесс оптимизации приводит к значительным сдвигам атомов от их стартовых положений. Геометрия ближайшего окружения примесных атомов, полученная по окончании процесса оптимизации, показана на рис. 2 и 3. Все данные позиции являются стабильными (метастабильными), поскольку им соответствуют глобальные (локальные) минимумы полной энергии.

Соответствующие оптимизированным позициям значения полной энергии сверхъячейки приведены в табл. 1. В данном случае мы пренебрегали возможностью появления вакансий в кислородной подрешетке.
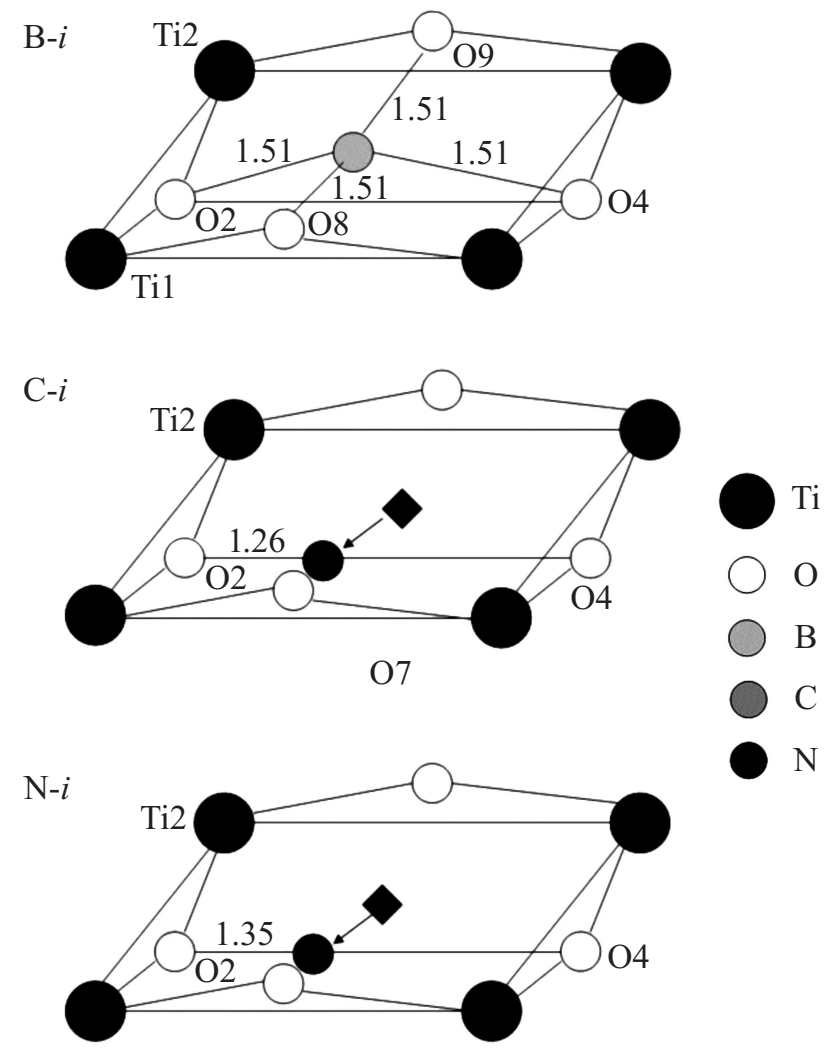

Рис. 3. Схема расположения атомов ближайшего окружения вокруг примесных атомов вокруг позиции $i$. Показаны длины связей между соседними атомами, в ед. А.. 
Таблица 1. Значения полной энергии сверхъячейки $E_{\mathrm{tot}}$ для различных позиций примесных атомов

\begin{tabular}{l|c|c|c}
\hline \multicolumn{1}{c|}{ Система } & $E_{\text {tot }}\left(A_{i}\right), \mathrm{eV}$ & $E_{\text {tot }}\left(A_{i 2}\right), \mathrm{eV}$ & $E_{\text {tot }}\left(A_{i 2}\right)-E_{\text {tot }}\left(A_{i}\right), \mathrm{eV}$ \\
\hline $\begin{array}{l}\text { Внедрение } \\
\begin{array}{l}\text { бора } \\
\text { Внедрение }\end{array}\end{array}$ & -430.55 & -431.05 & -0.50 \\
$\begin{array}{l}\text { углерода } \\
\text { Внедрение } \\
\text { азота }\end{array}$ & -428.32 & -428.64 & -0.32 \\
& & -427.12 & 1.26
\end{tabular}

Из данных табл. 1 по значениям величины $E_{\mathrm{tot}}\left(A_{i 2}\right)-E_{\mathrm{tot}}\left(A_{i}\right)$ в случае допирования бором и углеродом можно ожидать, что при термодинамическом равновесии предпочтительной является позиция $i 2$, тогда как при допировании азотом предпочтительной является позиция $i$, с короткой $\mathrm{N}-\mathrm{O}-$ связью. Видно также, что для конфигурации с минимальной энергией характер ближайшего окружения при переходе от бора к углероду и азоту изменяется, что связано с их длинами связей. Так атом бора связан короткими связями с тремя соседними атомами кислорода длиной от 1.37 до $1.40 \AA$. В свою очередь атом углерода образует две короткие связи с кислородом с длинами $1.39 \AA$ и углом между ними равным $112^{\circ}$. Атом азота образует только одну короткую связь с соседним атомом кислорода длиной $1.35 \AA$, направленную вдоль прямой, соединяющей атомы кислорода в соседних ячейках.

Ранее геометрия локального окружения примесных атомов бора рассматривалась в работах $[18,24,30]$ на основе данных из расчетов методом PW с ультрамягкими псевдо-потенциалами (реализован в программах Quantum Espresso и Castep). Авторы обеих работ пришли к выводу, что оптимальным местом локализации атома бора, внедренного в междоузлие, является положение внутри треугольника из атомов кислорода. Согласно работе [30], длины $\mathrm{B}-\mathrm{O}-$-вязей в данном треугольнике равны 1.38 и $1.39 \AA$, а согласно работе [18] соответственно 1.37 и $1.41 \AA$, что хорошо соответствует нашим данным, 1.38 и $1.40 \AA \AA$. Относительно локального окружения атома бора, замещающего атом кислорода, также имеется хорошее соответствие между нашими данными и предыдущими результатами: согласно [30], длины связей между атомом бора и ближайшими атомами Ті равны 2.12 и $2.38 \AA$, а согласно нашим данным 2.08 и $2.42 \AA$ соответственно.

Локальное окружение примесных атомов углерода в анатазе ранее изучалось на базе PW-расчетов в работе [31]. В случае замещения атомом углерода атома кислорода имеется заметное различие между нашими расчетными длинами Ті-С-связей и полученными в [31]. По нашим данным, эти длины связей равны 1.91 и $2.24 \AA$, а по данным [31] они составляют 2.081 и $2.21 \AA$. Согласно [31], при внедрении атома углерода в междоузлие он образует связи почти одинаковой длины, от 1.415 до $1.432 \AA$ с тремя соседними атомами кислорода (соответствующим атомам O1, O3, O7 на рис. 1). По нашим данным, в этом случае имеются только две связи с длиной $1.40 \AA$. Мы полагаем, что более правильным является наш вывод, поскольку примененный нами расчетный метод PAW является более точным и пригодным для химических соединений с произвольной степенью ионности.

Оптимизация геометрии кристалла вокруг внедренных в анатаз атомов азота была выполнена в работе [26]. Полученные авторами данные по длинам связи в случае замещения кислорода, 1.96 и $2.08 \AA$, хорошо соответствуют нашим данным, 1.92, 1.97 и $2.05 \AA$. Вывод авторов [26] о том, что в случае внедрения атома азота в междоузлие он образует одиночную связь с соседним атомом кислорода длиной $1.36 \AA$, также соответствует нашим результатам.

Заметим, что данный подход, основанный только на расчетах полной энергии, позволяет лишь сопоставить вероятности заселения атомами разных междоузельных позиций, но не сопоставить вероятности внедрения в междоузлия и замещения атомов кислорода. Согласно обсуждаемым ниже расчетам термодинамических характеристик образования дефектов, в условиях термодинамического равновесия и в отсутствии кислородных вакансий лишь позиция $i 2$ внедрения атома бора является стабильной. Позиции $i 2$ внедрения атома углерода при некоторых условиях и позиция $i$ внедрения азота являются метастабильными и могут быть заняты атомами при отсутствии термодинамического равновесия, которое может иметь место, например, при недостаточно длительном отжиге в процессе синтеза.

\section{4. Метод расчета термодинамических характеристик образования вакансий и допирования бором, углеродом, азотом}

В обзоре [32] было показано, что вклад колебательной энтропии в энергию образования точечных дефектов в полупроводниках не превышает $3-5 k_{B} T$, что соответствует при $T=900 \mathrm{~K}$ энергии $\sim 0.3 \mathrm{eV}$. Это значение на $\sim 4$ порядка меньше электронно-ядерной энергии ячейки кристалла. Кроме того, при расчетах энергии образования дефектов большая часть энтропийного вклада сокращается, поэтому при вычислении энергии образования дефектов в анатазе мы пренебрегали энтропийным вкладом и основывались лишь на первопринципных расчетах полной энергии сверхячеек $E_{\text {tot. }}$ В данном приближении свободная энергия образования кислородной вакансии в 48-атомной ячейке анатаза может быть записана как

$$
E_{f}(\mathrm{vac})=E_{\mathrm{tot}}\left(\mathrm{Ti}_{16} \mathrm{O}_{31}\right)+\mu(\mathrm{O})-E_{\text {tot }}\left(\mathrm{Ti}_{16} \mathrm{O}_{32}\right)
$$

Здесь $\mu(\mathrm{O})$ - химимческий потенциал (ХП) атома кислорода. Аналогично, если в ячейку с вакансией в кис- 
лородной подрешетке дополнительно введена примесь замещения $A_{\mathrm{O}}, A=B \vee C \vee N$, то энергия образования вакансии может быть вычислена как

$$
E_{f}\left(\operatorname{vac}, A_{\mathrm{O}}\right)=E_{\text {tot }}\left(\mathrm{Ti}_{16} \mathrm{O}_{30} A_{\mathrm{O}}\right)+\mu(\mathrm{O})-E_{\text {tot }}\left(\mathrm{Ti}_{16} \mathrm{O}_{30} A_{\mathrm{O}}\right) \text {, }
$$

а при внедрении атома в междоузлие $I=i 2 \vee i-$ как

$$
E_{f}\left(\operatorname{vac}, A_{I}\right)=E_{\mathrm{tot}}\left(\mathrm{Ti}_{16} \mathrm{O}_{31} A_{I}\right)+\mu(\mathrm{O})-E_{\mathrm{tot}}\left(\mathrm{Ti}_{16} \mathrm{O}_{30} A_{I}\right) \text {. }
$$

Заметим, что данные величины можно интерпретировать и как энергии образования примесей бора, углерода или азота в присутствии кислородных вакансий.

Ниже мы полагаем, что ХП атома кислорода $\mu(\mathrm{O})=\mu\left(\mathrm{O}_{2}\right) / 2$. ХП молекулы кислорода $\mu\left(\mathrm{O}_{2}\right)$ зависит от температуры и парциального давления кислорода в реакционной среде [33]; в его расчетах мы учли данные зависимости. Стандартным образом химпотенциал определен как потенциал Гиббса в расчете на одну молекулу, $\mu=d G(p, V) / d N$, а $d G=-S d t+V d p$. Применяя модель идеального газа, для которого $p V=N k_{B} T$, получаем

$$
\mu(T, p)=G\left(T, p_{0}\right)+\Delta \mu(T, p),
$$

где

$$
\Delta \mu(T, p)=k_{B} T \ln \left(p / p_{0}\right),
$$

a $G\left(T, p_{0}\right)$ - потенциал Гиббса при стандартном давлении $(1 \mathrm{~atm})$ и температуре реакции. При предельно высокой концентрации молекул, т. е. при их конденсации, и предельно низких температурах можно считать, что $G\left(0, p_{\text {cond }}\right)=E_{\text {tot }}$, где энергия молекулы кислорода $E_{\text {tot }}$ должна быть вычислена в рамках тех же приближений, которые были использованы в расчетах твердого тела. Конденсация кислорода при комнатной температуре происходит при давлении $\sim 9 \mathrm{GPa}$, а обычным давлением при синтезе является близкое к атмосферному или ниже, поэтому в случае кислорода вводим для этой величины поправку $-0.88 \mathrm{eV}$, которая соответствует величине $\Delta \mu(T, p)$ в уравнении (5) при $T=900 \mathrm{~K}$. Учитывая сказанное, скорректированное значение

$$
G\left(0, p_{0}\right)=E_{\text {tot }}-0.88=\mu_{\mathrm{el}}
$$

мы именуем электронной частью ХП.

Аналогичным образом мы оценивали ХП молекул $\mathrm{BO}, \mathrm{CO}, \mathrm{NO}$, необходимый для расчета энергии окисления примесей в кислородном окружении - см. ниже. Какие-либо данные о конденсации молекул ВО, $\mathrm{CO}$, NO при высоком давлении и температуре выше комнатной отсутствуют. Поэтому для данных молекул, ввиду близости их экспериментальных термодинамических характеристик к характеристикам молекулярного кислорода [34], применялось то же значение поправки, $-0.88 \mathrm{eV}$. Тогда полный ХП может быть вычислен как

$$
\mu(T, p)=\mu_{\mathrm{el}}+\Delta \mu\left(T, p_{0}\right)+\Delta \mu(T, p) .
$$

Таблица 2. Значения вкладов в ХП молекул $\mathrm{O}_{2}, \mathrm{BO}, \mathrm{CO}$ и NO, а также в суммарные ХП для температуры $T=900 \mathrm{~K}$ и давления $p_{0}=1 \mathrm{~atm}$ и $p_{\text {vac }}=10^{-30} \mathrm{~atm}(\mathrm{eV})$. (Приведены также суммарные значения ХП при давлениях $p_{0}$ и $\left.p_{\mathrm{vac}}\right)$

\begin{tabular}{c|c|c|c|l}
\hline Система & $\mathrm{O}_{2}$ & $\mathrm{BO}$ & $\mathrm{CO}$ & $\mathrm{NO}$ \\
\hline$\mu_{\mathrm{el}}$ & -10.72 & -11.45 & -15.65 & -12.08 \\
$\Delta \mu\left(T, p_{0}\right)$ & -1.74 & -1.92 & -1.88 & -1.98 \\
$\Delta \mu(T, p)$ & -5.35 & 5.35 & -5.35 & -5.35 \\
$\mu\left(T, p_{0}\right)$ & -12.56 & -17.53 & -17.53 & -14.06 \\
$\mu\left(T, p_{\text {vac }}\right)$ & -17.86 & -22.83 & -22.83 & -19.36
\end{tabular}

Здесь

$\Delta \mu\left(T, p_{0}\right)=\left[H\left(T, p_{0}\right)-H\left(0, p_{0}\right)\right]-T\left[S\left(T, p_{0}\right)-S\left(0, p_{0}\right)\right]$

— температурно-зависящий вклад в ХП при нормальном давлении, в котором $H$ и $S-$ соответственно энтальпия и энтропия в пересчете на одну молекулу. При их расчете были использовали данные из работы [34].

Мы полагаем, что температура $T=900 \mathrm{~K}$, что является типичным для отжига в процессе синтеза, а парциальное давление кислорода может изменяться от $1 \mathrm{~atm}$ до $10^{-30} \mathrm{~atm}$ (последнее - условный вакуум). Данные условия рассматриваем как крайние, между которыми зависящий от давления вклад в ХП изменяется от 0 до $-5.35 \mathrm{eV}$. Вычисленные таким образом значения ХП приведены в табл. 2.

Данная методика расчета позволяет оценить энергию образования кислородных вакансий как в чистом, так и в допированном анатазе. Метод расчета энергии образования примесных атомов в конденсированной среде можно так же определить аналогичным образом. А именно, для примеси замещения $A_{\mathrm{O}}$ энергия образования примесного центра будет

$$
E_{f}\left(A_{\mathrm{O}}\right)=E_{\text {tot }}\left(\mathrm{Ti}_{16} \mathrm{O}_{31} A_{\mathrm{O}}\right)+\mu(\mathrm{O})-\left[E_{\text {tot }}\left(\mathrm{Ti}_{16} \mathrm{O}_{32}\right)+\mu(A)\right],
$$

а для примеси внедрения атома $A=B \vee C \vee N$ в междоузельное положение $I=i_{2} \vee i$

$$
E_{f}\left(A_{I}\right)=E_{\text {tot }}\left(\mathrm{Ti}_{16} \mathrm{O}_{30} A_{I}\right)-\left[E_{\text {tot }}\left(\mathrm{Ti}_{16} \mathrm{O}_{32}\right)+\mu(A)\right] .
$$

Однако, попытки вычислить энергию образования примесных атомов осложнен тем, что основным методом введения примесей $\mathrm{B}, \mathrm{C}, \mathrm{N}$ является прекурсорный синтез, при котором ХП $\mu(A)$ остается неопределенным. Однако можно вычислить так называемую энергию предпочтения $E_{p r}$ междоузельной позиции I - величину, свободную от неопределенного ХП $\mu(A)$, которую определяем как [29]

$$
E_{p r}\left(A_{I}\right)=E_{f}\left(A_{I}\right)-E_{f}\left(A_{\mathrm{O}}\right) .
$$

Ниже покажем, что значения этой величины достаточно велики и указывают на наиболее вероятные позиции, которые могут занимать допирующие атомы в кристалле. 
Выражения для $E_{p r}$ можно записать в виде

$$
\begin{gathered}
E_{p r}\left(B_{i_{2}}\right)=E_{\text {tot }}\left(\mathrm{Ti}_{16} \mathrm{O}_{32} B_{i_{2}}\right)-E_{\text {tot }}\left(\mathrm{Ti}_{16} \mathrm{O}_{31} B_{\mathrm{O}}\right)-\mu(\mathrm{O}), \\
E_{p r}\left(A_{I}\right)=E_{\text {tot }}\left(\mathrm{Ti}_{16} \mathrm{O}_{32} A_{I}\right)-E_{\text {tot }}\left(\mathrm{Ti}_{16} \mathrm{O}_{31} A_{\mathrm{O}}\right)-\mu(\mathrm{O}),
\end{gathered}
$$

$A=C \vee N, I=i 2$ для С и $I=i$ для $\mathrm{N}$.

На основании первопринципных квантово-химических расчетов и оценок ХП можно также определить стабильность примесей в анатазе в кислородном окружении. Прежде всего, исходим из того, что внедренные в структуру анатаза примеси могут реагировать с молекулами кислорода, что может приводить к ликвидации примесного центра и образованию молекул ВО, СО и NO в кислородном окружении. Для примесей замещения, $A=C \vee N$, и внедрения, $A=B$, соответствующие уравнения имеют вид $\mathrm{Ti}_{16} \mathrm{O}_{31} A_{\mathrm{O}}+\mathrm{O}_{2} \rightarrow \mathrm{Ti}_{16} \mathrm{O}_{32}+A \mathrm{O}$ и $\mathrm{Ti}_{16} \mathrm{O}_{32} A_{i 2}+\mathrm{O} \rightarrow \mathrm{Ti}_{16} \mathrm{O}_{32}+A \mathrm{O}$. Для описания данных процессов мы вводим понятие энергии окисления примеси $E_{\text {ox }}$

$$
\begin{aligned}
E_{\mathrm{ox}}\left(A_{\mathrm{O}}\right)= & E_{\mathrm{tot}}\left(\mathrm{Ti}_{16} \mathrm{O}_{32}\right)+\mu(A \mathrm{O}) \\
& -E_{\mathrm{tot}}\left(\mathrm{Ti}_{16} \mathrm{O}_{31} A_{\mathrm{O}}\right)-2 \mu(\mathrm{O})
\end{aligned}
$$

и

$$
\begin{aligned}
E_{\text {ox }}\left(A_{i 2}\right)= & E_{\text {tot }}\left(\mathrm{Ti}_{16} \mathrm{O}_{32}\right)+\mu(A \mathrm{O}) \\
& -E_{\text {tot }}\left(\mathrm{Ti}_{16} \mathrm{O}_{32} A_{i 2}\right)-\mu(\mathrm{O}) .
\end{aligned}
$$

На основе расчетов данных величин ниже продемонстрируем, что примеси углерода или азота в анатазе в отсутствии кислородных вакансий являются термодинамически нестабильными.

\section{5. Результаты расчетов термодинамических характеристик и их обсуждение}

В табл. 3 показаны результаты расчетов $E_{p r}$ примесными атомами междоузельных позиций. Приведены значения величин $E_{p r}^{1}$, соответствующие учету только электронной части ХП $\mu_{\mathrm{el}}$, величин $E_{p r}^{2}-$ учет двух вкладов в ХП $\mu_{\mathrm{el}}+\Delta \mu\left(T, p_{0}\right)$, и $E_{p r}^{3}-$ при учете полного ХП $-\mu_{\mathrm{el}}+\Delta \mu\left(T, p_{0}\right)+\Delta \mu(p, T)$.

Для случая допирования бором были проведены тестовые расчеты энергии предпочтения при увеличении максимальной энергии плоских волн до $500 \mathrm{eV}$, а числа волновых векторов в НЧЗБ до 112. Полученное значение энергии предпочтения было равным $8.51 \mathrm{eV}$, что указывает на корректность выбора данных параметров расчетов.

Ранее в работах $[23,24,26]$ были выполнены расчеты энергии образования примесей бора, углерода и азота в анатазе. В табл. 3 также приведены энергии предпочтения, вычисленные нами, исходя из результатов данных работ. Видно, что на качественном уровне наши результаты согласуются с результатами указанных работ. Имеющиеся в некоторых случаях отклонения
Таблица 3. Значения энергии предпочтения междоузельных позиций $E_{p r}\left(A_{I}\right)$ (в $\mathrm{eV}$ ) для анатаза, допированного бором, углеродом, азотом. (В скобках приведены значения энергии предпочтения, полученные нами, исходя из энергии образования дефектов, вычисленной в работах $[23,24,26])$

\begin{tabular}{l|c|c|c}
\hline \multirow{2}{*}{\multicolumn{1}{c|}{ Система }} & \multicolumn{3}{|c}{$E_{p r}$, допирование } \\
\cline { 2 - 4 } & $\begin{array}{c}\text { бором } \\
(\mathrm{eV})\end{array}$ & $\begin{array}{c}\text { углеродом } \\
(\mathrm{eV})\end{array}$ & $\begin{array}{c}\text { азотом } \\
(\mathrm{eV})\end{array}$ \\
\hline$E_{p r}^{1}-$ учет $\mu_{\mathrm{el}}$ & -8.52 & -3.06 & -0.27 \\
$E_{p r}^{2}-$ учет & -7.46 & -2.1 & 0.68 \\
$\mu_{\mathrm{el}}+\Delta \mu\left(T, p_{0}\right)$ & $(-7.6)$ & $(-3.4)$ & $(0.0)$ \\
$E_{p r}^{3}-$ учет & -4.91 & 0.46 & 3.34 \\
$\mu_{\mathrm{el}}+\Delta \mu\left(T, p_{0}\right)+\Delta \mu(p, T)$ & $(-4.2)$ & 0.3 & $(2.4)$
\end{tabular}

связаны с различиями в способах оценки ХП и, вероятно, с погрешностями в расчетах полной энергии, присущими расчетному методу $\mathrm{PW}$ с ультрамягкими псевдопотенциалами.

Из полученных результатов для случая допирования бором следует, что при любых температуре и давлении кислорода в отсутствии кислородных вакансий следует ожидать, что предпочтительной позицией атомов бора будет междоузельная позиция $i 2$. В случае допирования углеродом синтез при высоких температурах и давлении кислорода $\sim 1$ atm может привести к появлению углерода в междоузельных позициях $i 2$, тогда как синтез при низких давлениях - к тому, что более выгодным может стать присутствие атомов углерода в позициях атомов кислорода; т.е. при низких давлениях кислорода, в частности, при синтезе в атмосфере инертного газа, присутствие атомов углерода возможно в обоих типах позиций. В случае допирования азотом из положительных значений величин $E_{p r}^{2}$ и $E_{p r}^{3}$ следует ожидать, что предпочтительными позициями атома азота будут позиции замещенных атомов кислорода при синтезе как в условиях высоких температур и высоких давлений кислорода, так и при высоких температурах и низких давлениях.

На качественном уровне данная закономерность коррелирует с характером локального окружения примесного атома: высокой стабильности атомов бора в междоузельной позиции соответствует образование им коротких связей с тремя соседними атомами, тогда как понижение стабильности атомов углерода и азота в междоузельной позиции является следствием понижения числа их связей с соседями.

Основным экспериментальным методом, который используется как средство идентификации местоположений примесных атомов, является метод рентгеновской эмиссионной спектроскопии. В случае допирования бором большинство исследователей приходят к выводу о том, что атомы бора внедряются в междоузлия $[17,20,35,36]$. Присутствие атомов бора в междоузлиях идентифицируется по наличию полосы в $B 1 s$-спектрах с энергией около $192 \mathrm{eV}$. Аналогично, 
присутствие полосы в $C 1 s$-спектрах при энергии около $282 \mathrm{eV}$, характерной для спектров карбида титана, позволяет идентифицировать присутствие атомов углерода в позиции замещения кислородных атомов [37]. Присутствие атомов азота, замещающих атомы кислорода, также подтверждается $N 1 s$-спектрами, в которых в данном случае имеется полоса с энергией вблизи $396 \mathrm{eV}$, характерная для нитрида титана [26], т.е. результаты наших расчетов разумно соответствуют данным предыдущих теоретических работ и имеющимся экспериментальным данным. Однако существенным обстоятельством является то, что в кислородной атмосфере внедренные в анатаз примеси могут удаляться, образуя молекулы ВО, $\mathrm{CO}$ и NO. Мерой стабильности примесей относительно данных процессов является величина их энергии окисления. Ее значения, вычисленные согласно (14) и (15) для атмосферного давления и вакуума, приведены в табл. 4.

Из данных таблицы следует, что лишь примеси бора при низком давлении могут быть стабильными. Отрицательные значения энергии окисления примесей углерода и азота свидетельствуют о термодинамической нестабильности данных примесей относительно взаимодействия с кислородом при любом давлении кислорода. Отсюда следует предположение о том, что при синтезе в кислородной атмосфере внедрение примесей атомов углерода и азота в условиях термодинамической стабильности, вероятно, является возможным в сочетании с кислородными вакансиями. Степень стабильности примесей в присутствии вакансий можно оценить, исходя из значений энергии образования вакансий, которая при наличии допирующих атомов является и энергией допирования в присутствии вакансий.

Таблица 4. Значения энергии окисления примесей $E_{\text {ох }}($ в $\mathrm{eV})$ в анатазе для атмосферного давления кислорода и в условиях вакуума при температуре $900 \mathrm{~K}$

\begin{tabular}{l|r|c|c}
\hline \multirow{2}{*}{ Система } & \multicolumn{3}{|c}{ Примесь } \\
\cline { 1 - 4 } & бора $B_{i 2}$ & углерода С & азота $\mathrm{N}_{\mathrm{O}}$ \\
\hline Давление $p=1 \mathrm{~atm}$ & -2.24 & -11.35 & -6.10 \\
Давление $p=10^{-30} \mathrm{~atm}$ & 0.45 & -5.45 & -0.80
\end{tabular}

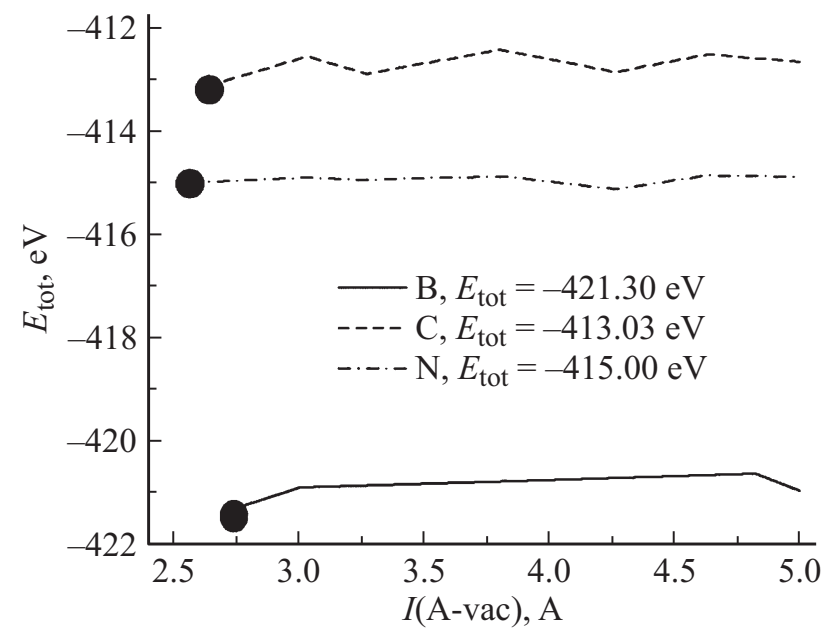

Рис. 4. Зависимости полной энергии от расстояния между примесными атомами и вакансиями (точками отмечены соответствующие минимальные значения полной энергии). В вставке приведены значения полных энергий для указанных точек.

В случае одной вакансии на сверхъячейку значения полной энергии $E_{\text {tot }}$ непосредственно получаются из расчетов в результате оптимизации структуры. Однако, если в ячейке присутствуют примесный атом и вакансия, то оптимизированная полная энергия сверхъячейки зависит от расстояния между ними. В расчетах энергии образования вакансий в качестве величины $E_{\text {tot }}$ мы использовали минимальное ее значение, которое получали путем построения зависимости $E_{\text {tot }}$ от расстояния между примесными атомами и вакансией. Поскольку координаты вакансии точно не определены, под данным расстоянием понимали расстояние между центрами примесного атома и атома, который удалялся при образовании вакансии. Полученные зависимости приведены на рис. 4, вместе с минимальными значениями $E_{\text {tot }}$. Минимальные значения $E_{\text {tot }}$ соответствуют расстояниям между атомами и вакансиями от 2.5 до $2.8 \AA$.

В табл. 5 представлены значения энергии образования вакансий при $T=900 \mathrm{~K}$, атмосферном давлении и в вакууме, вычисленные как в отсутствии примесных атомов, так и при их наличии. Показаны значения величин

Таблица 5. Значения энергии образования вакансий $E_{f}(\mathrm{vac})(\mathrm{B} \mathrm{eV})$ в чистом анатазе и допированном атомами бора, углерода, азота. (В скобках приведены значения энергии образования вакансий, полученные в работах $[23,24,26]$ )

\begin{tabular}{|c|c|c|c|c|}
\hline \multirow{2}{*}{ Система } & \multirow{2}{*}{$\begin{array}{c}E_{f}(\mathrm{vac}) \\
\text { без допирования }\end{array}$} & \multicolumn{3}{|c|}{$E_{f}(\mathrm{vac})$, допирование } \\
\hline & & бором & углеродом & азотом \\
\hline$E_{f}^{1}(\mathrm{vac})-$ учет $\mu_{\mathrm{el}}$ & $\begin{array}{r}4.08 \\
(4.2)\end{array}$ & $\begin{array}{r}5.02 \\
(4.6)\end{array}$ & $\begin{array}{l}1.80 \\
(1.9)\end{array}$ & $\begin{array}{r}1.96 \\
(0.6)\end{array}$ \\
\hline$E_{f}^{2}(\operatorname{vac})-$ учет $\mu_{\mathrm{el}}+\Delta \mu\left(T, p_{0}\right)$ & 3.12 & $\begin{array}{r}4.06 \\
(5.3)\end{array}$ & $\begin{array}{l}0.84 \\
(1.5)\end{array}$ & $\begin{array}{r}0.97 \\
(1.0)\end{array}$ \\
\hline$E_{f}^{3}(\operatorname{vac})-$ учет $\mu_{\mathrm{el}}+\Delta \mu\left(T, p_{0}\right)+\Delta \mu(p, T)$ & 0.47 & $\begin{array}{r}1.41 \\
(1.7)\end{array}$ & $\begin{array}{l}-1.81 \\
(-2.1)\end{array}$ & $\begin{array}{l}-1.67 \\
(-1.8)\end{array}$ \\
\hline
\end{tabular}



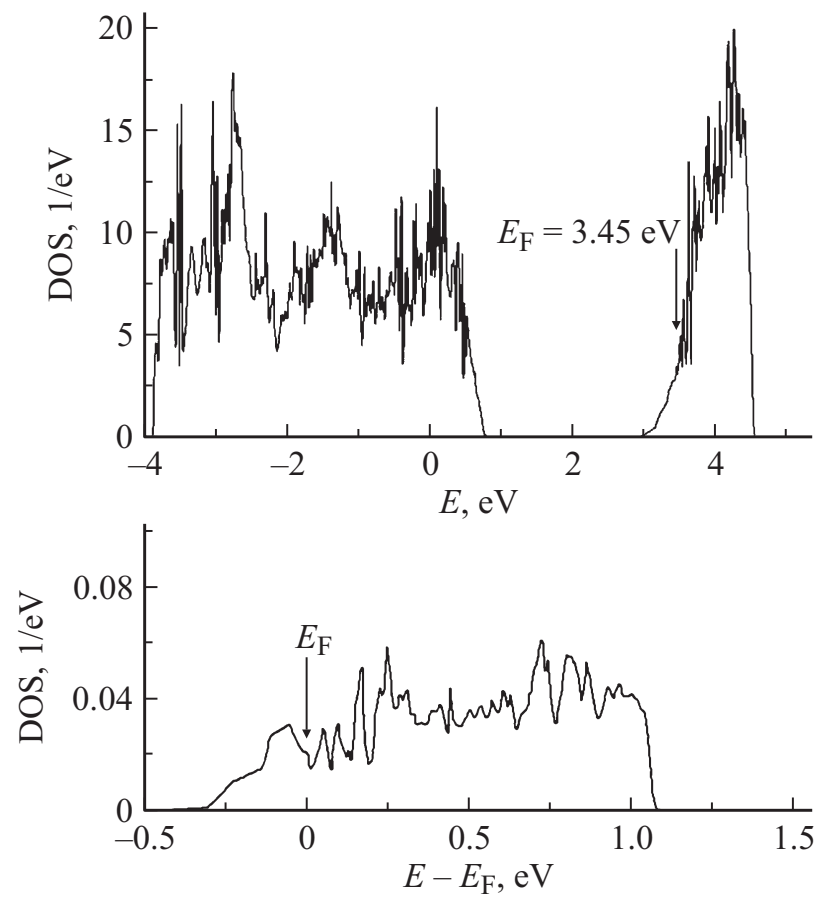

Pис. 5. ПС для анатаза, допированного бором. На верхней панели - полная ПС для атома бора в позиции $i 2$. На нижней панели - парциальная ПС атома бора.

$E_{f}^{1}(\mathrm{vac})$, соответствующие учету только электронной части ХП, $\mu_{\mathrm{el}}$, величин $E_{f}^{2}(\mathrm{vac})-$ учет двух вкладов в $\mathrm{XП} \mu_{\mathrm{el}}+\Delta \mu\left(T, p_{0}\right)$ и $E_{f}^{3}(\mathrm{vac})-$ при учете полного ХП $\mu_{\mathrm{el}}+\Delta \mu\left(T, p_{0}\right)+\Delta \mu(p, T)$. Предполагалось, что примесный атом бора занимает междоузельную позицию $i 2$, а атомы углерода и азота замещают атомы подрешетки кислорода.

Большое положительное значение $E_{f}^{1}(\mathrm{vac})$ „без допирования" свидетельствует о том, что при высокой концентрации кислорода и низкой температуре образование кислородных вакансий является маловероятным процессом. Из значений величин $E_{f}^{2}(\mathrm{vac})$ и $E_{f}^{3}(\mathrm{vac})$ „без допирования“ следует, что повышение температуры, а также понижение парциального давления кислорода приводит к существенному понижению энергии образования вакансий, но ее значение остается положительным, что соответствует малой вероятности образования вакансий. Результаты расчетов для случая допирования бором приводят к аналогичным выводам о малой вероятности образования вакансий при любых условиях, но ситуация изменяется при допировании углеродом или азотом. Значения энергии образования, хотя они и заметно меньше, чем для случаев „без допирования“ и допирования бором, остаются положительными для высокой концентрации кислорода, но при снижении парциального давления кислорода эти значения переходят в отрицательную область, что соответствует высокой вероятности появления в структуре анатаза допирующего атома в присутствии кислородной вакансии.
В литературе имеются результаты расчетов методом PW энергии образования вакансий в чистом и допированном бором, углеродом и азотом анатазе [23,24,26]. Эти данные, приведенные в табл. 5, разумно соответствуют нашим значениям, за исключением результата работы [26] по допированию азотом, $0.6 \mathrm{eV}$. Bсе расчеты выявляют тенденцию, заключающуюся в том, что допирование углеродом или азотом, но не бором, способствуют появлению в структуре анатаза кислородных вакансий. Данные результаты могут быть интерпретированы и как свидетельство того, что присутствие кислородных вакансий способствует образованию термодинамически стабильных примесей углерода или азота.

\section{6. Электронная зонная структура анатаза с примесями и вакансиями. Сравнение с экспериментом}

Выполненные расчеты электронной структуры с разрешенной спиновой поляризацией не во всех случаях привели к спин-поляризованому решению. В частности, сошлись к спин-ограниченному решению, т.е. к отсутствию магнитных моментов (расчеты для допирования бором при положении атомов в позициях $i 2$ ). На рис. 5 показана плотность электронных состояний (ПС) для размещения атомов бора в этих позициях. Все состояния, принадлежащие атому бора, погружены вглубь зоны проводимости, не имеющей спиновую поляризацию и образованной в основном $3 d$-состояниями атомов титана. Отметим существенную особенность состояний атома бора, плотность которого в прифермиевской области также показана на рис. 5. А именно, данная ПС составляет лишь весьма незначительную долю полной ПС, откуда следует, что атомы бора являются донорами электронов, отдающими их в зону проводимости, но слабо влияющими на плотность состояний в зоне. Вычисленная плотность состояний весьма близка к аналогичной ПС, вычисленной авторами работы [24]. В обоих расчетах зонные состояния бора погружены в зону проводимости, а ширина запрещенной зоны составляет $2.1 \mathrm{eV}$, что на $35 \%$ ниже экспериментального значения для чистого анатаза. Однако данные результаты качественно отличаются от результатов работы [18], согласно которым ширина запрещенной зоны в В-допированном анатазе должна составлять $9 \mathrm{eV}$, а состояния атома бора должны находиться внутри запрещенной зоны.

Использование имеющихся спектров ЭПР В-допированного анатаза [24] для проверки правильности нашей зонной структуры является весьма проблематичным, поскольку их интерпретация не является надежной. Однако корректность наших расчетов подтверждается экспериментальными спектрами оптического поглощения $[18,35]$, которые указывают на сдвиг края поглощения при В-допировании в голубую сторону, что соответствует сдвигу нашего уровня Ферми вглубь зоны проводимости. 
На рис. 6 приведена плотность состояний в С-допированном анатазе в отсутствие кислородных вакансий. Видно, что при таком допировании зонная структура является спин-поляризованной. Внутри запрещенной щели расположены 3 примесные зоны с положительной $z$-проекцией спина, принадлежащие атому углерода, а также 2 зоны с отрицательной проекцией спина, с энергией от 1.3 до $1.6 \mathrm{eV}$ и от 2.2 до $2.6 \mathrm{eV}$, тогда как третья примесная зона, с энергией от 2.6 до $2.9 \mathrm{eV}$, погружена в зону проводимости. Электронами заняты 3 зоны с $S_{z}=1 / 2$ и одна зона с $S_{z}=-1 / 2$, вследствие чего значение магнитного момента составляет 2 магнетона Бора. На рис. 7 показана полная ПС в С-допированном анатазе в присутствии кислородных вакансий. Видно, что появление кислородной вакансии приводит к заполнению двумя электронами состояний с $S_{z}=-1 / 2$, вследствие чего зонная структура становится не спин-поляризованной.

Однако данный вид зонной структуры не соответствует имеющимся данным по ЭПР-спектрам С-допированного анатаза [38-41], согласно общепринятой интерпретации которого спектр ЭПР относится к электронам в зоне вакансионных состояний, расположенных

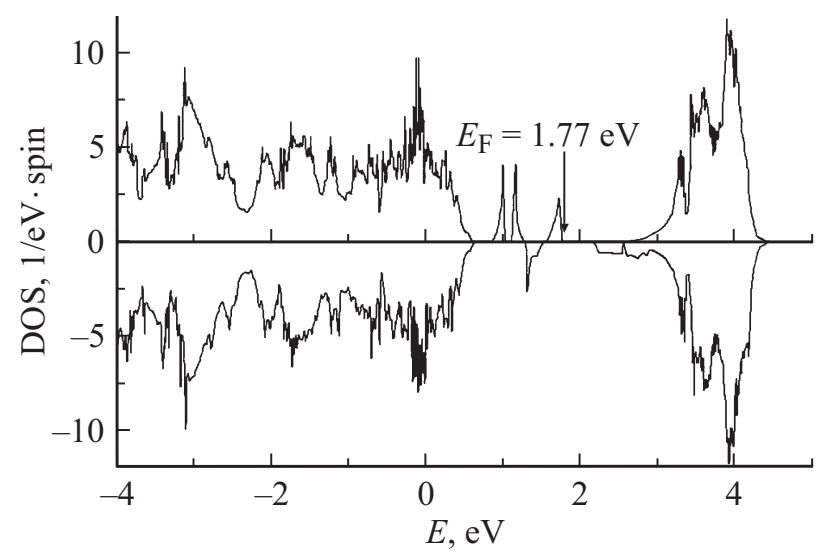

Рис. 6. ПС в С-допированном анатазе.

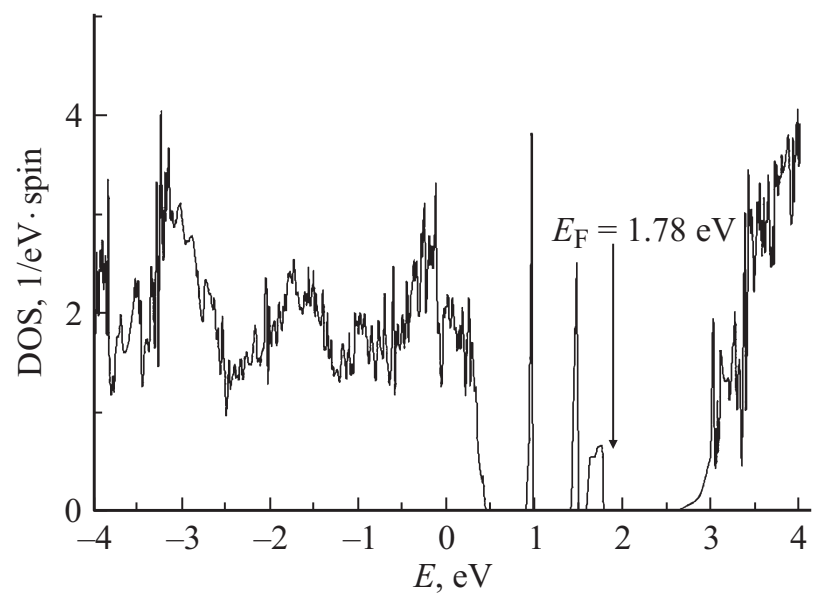

Рис. 7. ПС в С-допированном анатазе в присутствии кислородных вакансий.

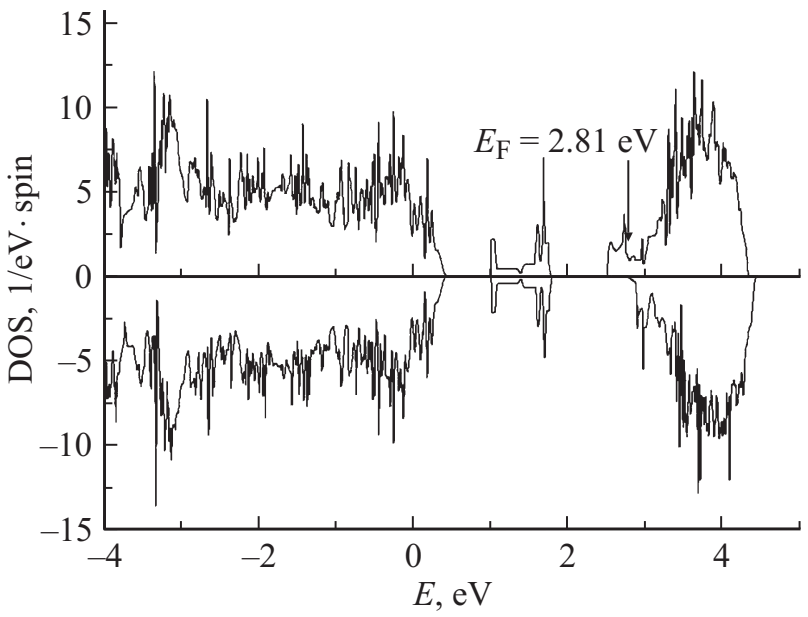

Рис. 8. ПС для С-допированного анатаза в присутствии двух кислородных вакансий.

вблизи потолка запрещенной зоны. Мы предположили, что спин-поляризованное состояние такого типа может быть реализовано при более высокой концентрации кислородных вакансий, и выполнили расчеты для нескольких вариантов расположения двух вакансий в суперьячейке. Один из них включал две вакансии, удаленные от атома углерода на максимальное расстояние, возможное в пределах суперъячейки, $5.45 \AA$, и на максимальное расстояние между ними, $10.24 \AA$. Три других варианта включали вакансии, расположенные на малом расстоянии от атома углерода, от 2.47 до $3.04 \mathrm{eV}$. Расчеты для вариантов с малыми расстояниями не привели к каким-либо решениям с вакансионными зонами, но вариант с максимальным расстоянием между вакансиями привел к нужному спин-поляризованному решению, плотность состояний для которого показана на рис. 8. В данном случае внутри запрещенной зоны, при энергии от 1.0 до $1.8 \mathrm{eV}$, имеются 6 занятых незначительно спин-поляризованных состояний атома углерода, а вблизи дна зоны проводимости, в диапазоне от 2.5 до $2.8 \mathrm{eV}$ - два занятых состояния с положительной $z$-проекцией спина. Суммарное значение магнитного момента (на ячейку) составляет 1.7 магнетона Бора, причем почти $70 \%$ магнитного момента относятся к ближайшим к вакансиям атомам титана. Таким образом, данные состояния можно считать состояниям вакансий (именуемыми также состояниями $\mathrm{Ti}^{3+}$ ), ответственным за появление ЭПР-спектров С-допированного анатаза.

На рис. 9 приведены ПС для $\mathrm{N}$-допированного анатаза в отсутствие кислородных вакансий и при их наличии. Расчеты при отсутствии вакансий приводят к спин-поляризованному решению. Рассчитанная ПС соответствует ранее полученным данным работы [26]. Спин-поляризованные состояния атома азота, с энергией от 0.6 до $1.05 \mathrm{eV}$, расположены внутри запрещенной зоны вблизи потолка валентной зоны. Имеется одна пустая зона с отрицательной проекцией спина вблизи $2 \mathrm{eV}$, т.е магнитный момент атома азота должен быть близок к 

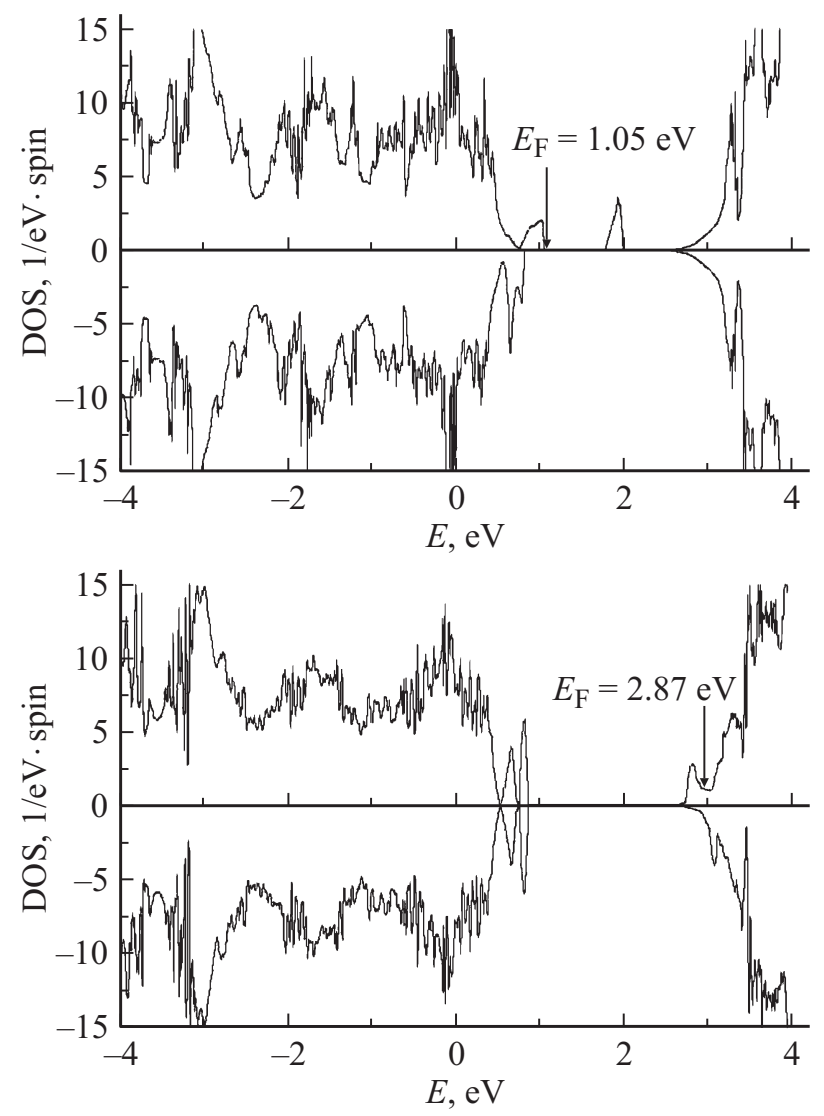

Pис. 9. ПС в N-допированном анатазе в отсутствии кислородных вакансий (верхняя панель) и при их наличии (нижняя панель).

1 магнетону Бора. Расчетное значение намагниченности составляет 0.69 магнетона Бора, из которых 0.49 приходится на атом азота, а 0.16 относятся к ближайшим к атому азота атомам кислорода, O1-O3-O5-O6 на рис. 1.

Однако и эта ПС не соответствует обычной интерпретации спектра ЭПР N-допированного анатаза [26], в которой спектр относят к наличию состояний кислородной вакансии. Приведенные на рис. 8 данные по ПС в N-допированном анатазе в присутствии вакансий подтверждают данную интерпретацию. Удаление кислородного атома приводит к переносу одного электрона на состояния атома азота, вследствие чего состояния этого атома теряют спиновую поляризацию. При этом из зоны проводимости опускается спин-поляризованное состояние с энергией от 2.5 до $2.9 \mathrm{eV}$, занятое одним электроном. Полная намагниченность ячейки составляет 0.71 магнетона, из которых 0.48 приходятся на 3 атома титана, ближайшие к кислородной вакансии, что соответствует имеющейся интерпретации спектра ЭПР.

Таким образом, учет присутствия вакансий в С- или $\mathrm{N}$-допированном анатазе приводит к виду электронной структуры, которая соответствует спектрам ЭПР. Это подтверждает вывод из предыдущего раздела о том, что наличие вакансий является фактором, способствующим стабилизации $\mathrm{C}$ - или $\mathrm{N}$-примесей при условии термодинамического равновесия.

\section{7. Заключение}

На основе расчетов электронной структуры методом PAW мы исследовали геометрию локального окружения примесей $\mathrm{B}, \mathrm{C}$ и $\mathrm{N}$ в анатазе. Было показано, что для атома В возможны 2 (стабильных или метастабильных) варианта размещения в междоузлиях кристаллической решетки с образованием трех и четырех связей с ближайшими атомами кислорода, а для атомов С и $\mathrm{N}$ - варианты с образованием одной и двух связей с соседними атомами кислорода. Минимальное значение полной энергии кристалла соответствует понижению числа локальных химических связей от трех для атома $\mathrm{B}$ до двух для атома С и одного для атома $\mathrm{N}$. C целью выявить места локализации примесей в условиях термодинамического равновесия установлены значения энергии предпочтения междоузельных позиций в сравнении с позициями замещения кислородных атомов. Было показано, что в случае В при любом давлении предпочтительной позицией является междоузельная с тремя связями с соседними атомами. Для случая допирования С при давлении кислорода близком к $1 \mathrm{~atm}$ предпочтительной является междоузельная позиция с двумя связями, но положительное значение энергии предпочтения при низком давлении свидетельствует о том, что более выгодной становится позиция замещения атомов кислорода. В случае допирования атомами $\mathrm{N}$ при любом давлении кислорода предпочтительной является позиция замещения атомов кислорода. Данная закономерность соответствует понижению от В к С числа локальных химических связей между междоузельными атомами и их ближайшем окружением.

В условии пребывания допированного анатаза в кислородной атмосфере существует вероятность удаления примесей из кристалла за счет их окисления с образованием молекул ВO, CO и NO. Была определена вероятность таких процессов путем расчета энергии окисления примесей при условии термодинамического равновесия. Показано, что лишь примеси В могут быть стабильными относительно таких процессов, тогда как примеси $\mathrm{C}$ и $\mathrm{N}$ нестабильны.

Исходя из зафиксированного в экспериментах факта пребывания в структуре анатаза кислородных вакансий, естественно предположить, что наличие вакансий может являться фактором, способствующим стабилизации примесей. Была дана оценка влияния кислородных вакансий на стабильность примесей $\mathrm{B}, \mathrm{C}$ и $\mathrm{N}$ путем расчета энергии образования кислородных вакансий и пар примесный атом-вакансия. Было показано, что при любом давлении кислорода присутствие вакансий не способствует стабилизации примесей В. Однако в случае допирования $\mathrm{C}$ или $\mathrm{N}$ наличие вакансий приводит к стабилизации примесных атомов. Отрицательные значения энергии образования пар примесный атом-вакансия 
при низком давлении кислорода свидетельствуют о высокой вероятности образования пар С-вакансия или $\mathrm{N}$-вакансия при термодинамическом равновесии.

Дан анализ полученные в расчетах спектров электронных состояний на предмет их соответствия данным ЭПР-спектроскопии, свидетельствующих о присутствии кислородных вакансий в допированном анатазе. В случае с углеродом или азотом экспериментальные данные показывают, что спектр ЭПР относится к электронам, находящимся в вакансионных состояниях вблизи потолка запрещенной зоны, что соответствует рассчитанной электронной структуре анатаза в случае пар $\mathrm{N}$-кислородная вакансия. В случае пар C-вакансия расчет показывает, что спин-поляризованный электрон может принадлежать атому углерода. Однако при более высокой концентрации кислородных вакансий было установлено, что электронные состояния, соответствующие интерпретации спектров ЭПР, при которой электроны с неспаренным спином занимают состояния, локализованы в основном на атомах, ближайших к кислородным вакансиям.

Приносим благодарность В.Н. Красильникову за полезные обсуждения.

\section{Список литературы}

[1] A. Fujishima, T. Rao, D. Tryk. J. Photochem. Photobiol. C 1, $1(2000)$.

[2] M.R. Hoffmann, S. Martin, W. Choi, D. Bahnemannt. Chem. Rev. 95, 69 (1995).

[3] K. Hashimoto, H. Irie, A. Fugishima. Jpn. J. Appl. Phys. 44, 8269 (2005).

[4] M. Kitano, M. Tsujimaru, M. Anpo. Top. Catal. 49, 4 (2008).

[5] R. Thakur, R. Chaudhary, C. Singh. Renewable Sustainable Energy 2, 042701 (2010).

[6] S. Gupta, M. Tripathi. Chinese Sci. Bull. 56, 1639 (2011).

[7] A. Zaleska. Rec. Patents Eng. 2, 157 (2008).

[8] В.Н. Красильников, А.П. Штин, О.И. Гырдасова, Е.В. Поляков, Л.Ю. Булдакова, М.Ю. Янченко, В.М. Зайнуллина, В.П. Жуков. Журнал неорган. химии 55, 1258 (2010).

[9] H. Irie, Y. Watanabe, K. Hashimoto. J. Phys. Chem. B 107, 5483 (2003).

[10] W. Ren, Zh. Ai, F. Jia, L. Zhang, X. Fan, Zh. Zou. Appl. Catal. 69, 138 (2007).

[11] S. Sakthivel, H. Kisch. Angew. Chem. Int. Ed. 42, 4908 (2003).

[12] R. Asahi, T. Morikawa, T. Ohwaki, K. Aoki, Y. Taga. Science 293, 269 (2001).

[13] S.-K. Joung, T. Amemiya, M. Murabayashi, K. Itoh. Chem. Europ. J. 12, 5526 (2006)

[14] S.-K. Joung, T. Amemiya, M. Murabayashi, K. Itoh. Appl. Catal. A 312, 20 (2006).

[15] H. Wang, J. Lewis. J. Phys. C 17, L209 (2005).

[16] M. Bettinelli, V. Dallacasa, D. Falcomer, P. Fornasiero, V. Gombac, T. Montini, L. Romany, A. Speghini. J. Hazard. Mater. 146, 529 (2007).

[17] D. Chen, D. Yang, Q. Wang, Z. Jiang. Ind. Eng. Chem. Res. 45, 4110 (2006).
[18] N. Feng, A. Zheng, Q. Wang, P. Ren, X. Gao, Sh.-B. Liu, Zh. Shen, T. Chen, F. Deng. J. Phys. Chem. C 115, 2709 (2011).

[19] C.-H. Wei, X.-H. Tang, J.-R. Liang, S.-Y. Tan. J. Environ. Sci. (China) 19, 90 (2007).

[20] W. Zhao, W. Ma, C. Chen, J. Zhao, Z. Shuai. J. Am. Chem. Soc. 126, 4782 (2004).

[21] В. Жуков, В. Зайнуллина, Е. Чулков. Перспективные фотокатализаторы. Электронная структура, оптические свойства и фотокаталитическая активность материалов на основе диоксида титана. Lambert Academic Publishing, Saarbrucken, Germany (2016).

[22] V. Zainullina, V. Zhukov, M. Korotinю. J. Photochem. Photobiology C 22, 58 (2015).

[23] C. DiValentin, G. Pacchioni, A. Selloni. Chem. Mater. 17, 6656 (2005).

[24] E. Finazzi, C.D. Valentin, G. Pacchioni. J. Phys. Chem. C 113, 220 (2009).

[25] C.D. Valentin, G. Pacchioni, A. Selloni. Phys. Rev. B 70, 085116 (2004).

[26] C.D. Valentin, A. Zheng, Q. Wang, P. Ren, X. Gao, Sh.-B. Liu, Zh. Shen, T. Chen, F. Deng. Chem. Phys. 339, 44 (2007).

[27] G. Kresse, M. Marsman, J. Furthmuller. http:/cms.mpi.univie.ac.at/vasp/guide/vasp.html (2011).

[28] G. Kresse, D. Joubert. Phys. Rev. B 59, 1758 (1999).

[29] V. Zhukov. Nanosystems: physics, chemistry, mathematics 5, 509 (2014).

[30] C. DiValentin, G. Pacchioni. Catal. Today 206, 12 (2011).

[31] C. DiValentin, G. Pacchioni, A. Selloni. Chem. Mater. 17, 6656 (2005).

[32] C.V. der Walle, J. Neugebauer. J. Appl. Phys. 95, 3851 (2004).

[33] K. Reuter, M. Scheffler. Phys. Rev. B 65, 035406 (2001).

[34] JANAF Thermochemical tables, volume NSRDS-NBS 37 / Eds D. Stull, H. Prophet. United States Department of Commerce and National Bureau of Standards, Office of Standard Reference Data, National Bureau of Standards, Washington, D.C., 20234 (1971).

[35] V. Gombac, L. Rogatis, A. Gasparotto, G. Vicario, T. Montini, D. Barreca, G. Balducci, P. Fornasiero, E. Tondello, M. Graziani. Chem. Phys. 339, 111 (2007).

[36] J. Yuan, E. Wang, Y. Chen, W. Yang, J. Yao, Y. Cao. Appl. Surf. Sci. 257, 7335 (2011).

[37] Y. Choi, T. Umebayashi, M. Yoshikawa. J. Mater. Sci. 39, 1837 (2004).

[38] E. Konstantinova, A. Kokorin, S. Sakthivel, H. Kisch, K. Lips. Chimia 61, 810 (2007).

[39] Z. Liu, R. Guo, G.R. Li, Q. Bu, W.X. Zhao, Y.X. Tong. Electrochim. Acta 59, 449 (2012).

[40] E.A. Reyes-Garcia, Y. Sun, K. Reyes-Gil, D. Raftery. Solid State Nucl. Magn. Res. 35, 74 (2009).

[41] Y. Wu, M. Xing, J. Zhang, F. Chen. Appl. Catal. B 97, 182 (2010). 